\title{
Custos de armadilhas adesivas artesanais para a captura de insetos-praga
}

\author{
Janaína Pereira dos Santos ${ }^{1}$, Everlan Fagundes² e Alexandre Carlos Menezes-Netto ${ }^{3}$
}

\begin{abstract}
Resumo - Armadilhas adesivas coloridas podem ser usadas no monitoramento e no controle de insetos-praga em cultivos agrícolas e em áreas urbanas. Neste trabalho calcularam-se os custos de dois modelos de armadilhas adesivas artesanais feitas com cola entomológica artesanal e comercial, em comparação a um modelo de armadilha comercial. Verificou-se que os modelos de armadilhas artesanais são mais viáveis economicamente, sendo 1,7 a 8,2 vezes mais baratos que o modelo comercial, proporcionando uma economia de até $\mathrm{R} \$ 1.429,07$ mensais ha-1.
\end{abstract}

Termos para indexação: Manejo integrado de pragas; Monitoramento; Controle.

\section{Costs of adhesive handcrafted traps for capturing insect pests}

Abstract - Colored adhesive traps can be used to monitor and control insect pests in agricultural crops and in urban areas. In this paper, the costs of two models of adhesive handcrafted traps made with handcrafted and commercial entomological glue were calculated, comparing to a commercial trap model. It was found that the models of handcrafted traps are more economically viable, being 1.7 to 8.2 times cheaper than the commercial model, providing savings of up to $\mathrm{R} \$ 1,429.07$ monthly ha-1.

Index terms: Integrated Pest Management; Monitoring; Control.

\section{Introdução}

No Manejo Integrado de Pragas (MIP) são utilizadas várias estratégias de controle que levam em consideração fatores que geram impactos sociais, econômicos e ambientais (KOGAN, 1998). Em cultivos agrícolas, onde se adotam os princípios do MIP, o monitoramento de insetos-praga com o auxílio de armadilhas é usado como informação-chave para a tomada de decisão de controle (SANTOS et al., 2008).

As armadilhas adesivas consistem em superfícies coloridas, geralmente amarelas ou azuis, impregnadas com uma substância aderente (RESENDE et al., 2007). O uso de armadilhas coloridas fundamenta-se no princípio de que os comprimentos de onda emitidos por algumas superfícies coloridas atraem diferentes espécies de insetos (PROKOPY \& BOLLER, 1971). Esse tipo de armadiIha pode ser usado no monitoramento e no controle de insetos-praga em lavouras, pomares, estufas, estábulos e até mesmo em áreas urbanas, como pomares domésticos, hortas caseiras e no interior de residências (SANTOS et al., 2017).

$\mathrm{O}$ uso de armadilhas adesivas de coloração amarela já foi relatado com sucesso na captura de vaquinhas (SANTOS et al., 2008), psilídeos (GUAJARÁ et al., 2004), cigarrinhas (MOLINA et al., 2010), pulgões (RESENDE et al., 2007; SANTOS et al., 2008), moscas-das-frutas (CYTRYNOWICZ et al., 1982), moscas-brancas (SANTOS et al., 2008), moscas-minadoras (SANTOS et al., 2008; GAERTNER \& BORBA, 2014) e moscas domésticas (KAVRAN et al., 2019). Já as armadilhas de coloração azul são eficientes na captura de moscas-dos-estábulos (CILEK, 2003) e tripes (GAERTNER \& BORBA, 2014).

Armadilhas adesivas coloridas são comercializadas na forma de placas ou rolos. Em algumas regiões do Brasil, a indisponibilidade de um local acessível para a compra, como casas agropecuá- rias por exemplo, aliada ao elevado custo são fatores que dificultam a aquisição desse tipo de artefato. Dessa forma, em 2016, na Epagri/Estação Experimental de Caçador (EECd), iniciou-se um estudo visando desenvolver armadilhas artesanais de baixo custo para a captura de insetos-praga.

Na primeira etapa do estudo foram desenvolvidos dois modelos de armadiIhas adesivas e uma cola entomológica artesanal para aderir os insetos nas armadilhas. Ao desenvolver os artefatos, preconizou-se o uso de materiais acessíveis, resistentes e de baixo custo, os quais foram confeccionados com garrafa PET de $500 \mathrm{~mL}$ e papel-cartão. Paralelamente, um estudo foi conduzido para avaliar a eficiência das armadilhas artesanais na captura de insetos. Os resultados foram abordados por SANTOS et al. (2017) e indicaram que os dois modelos artesanais foram eficientes e apresentaram captura similar ao comercial. Para o monitoramento de insetos,

Recebido em 11/8/2020. Aceito para publicação em 8/10/2020.

2 Engenheiro-agrônomo, Dr., e-mail: everlanf@gmail.com.

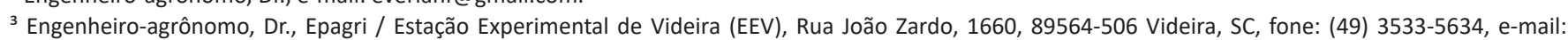
alexandrenetto@epagri.sc.gov.br. 
recomenda-se a utilização de 100 armadilhas artesanais/ha. Para o controle não há um número definido, entretanto, para proporcionar a captura massal dos insetos-praga, sugere-se instalar um maior número possível de armadilhas na área de cultivo.

Na segunda etapa, objeto do presente estudo, foram calculados os custos dos modelos de armadilhas artesanais em comparação ao modelo comercial do tipo placa adesiva. Os custos foram calculados considerando-se o uso de 100 armadilhas artesanais/ha, feitas com cola entomológica artesanal (de baixa durabilidade - 5 a 7 dias) e cola entomológica comercial (de alta durabilidade - superior a 30 dias). Para comparar os custos dos modelos artesanais com o comercial utilizaram-se os valores unitários. O orçamento dos materiais utilizados na confecção das armadilhas artesanais foi feito em agosto de 2020, em três locais distintos do comércio de Caçador, SC, tais como papelarias, supermercados, agropecuárias e lojas de materiais de construção. Para o cálculo dos custos, utilizou-se o valor médio dos três locais de cotação, devido à possibilidade de variação de valores entre os locais. Os orçamentos da cola entomológica e da armadilha comercial foram obtidos via internet com três fabricantes diferentes, sem considerar o valor do frete.

\section{Modelos de armadilhas artesanais desenvolvidos pela Epagri/EECd}

Modelo A: confeccionado com recipiente plástico descartável, tipo garrafa PET, com capacidade para $500 \mathrm{~mL}$. As embalagens vazias foram pintadas internamente com tinta a óleo de coloração amarela (tonalidade "Ouro") ou azul-escuro (tonalidade "Del Rey") (Figura 1). A cola entomológica (artesanal ou comercial) foi aplicada com um pincel na parte externa da garrafa. Para pendurar as armadilhas fez-se um orifício na tampa da garrafa e fixou-se um pedaço de arame fino de aproximadamente $20 \mathrm{~cm}$.

Modelo B: confeccionado com foIhas de papel-cartão $(66 \mathrm{~cm} \times 48 \mathrm{~cm})$ amarelo e azul-escuro (Figura 1), colori-

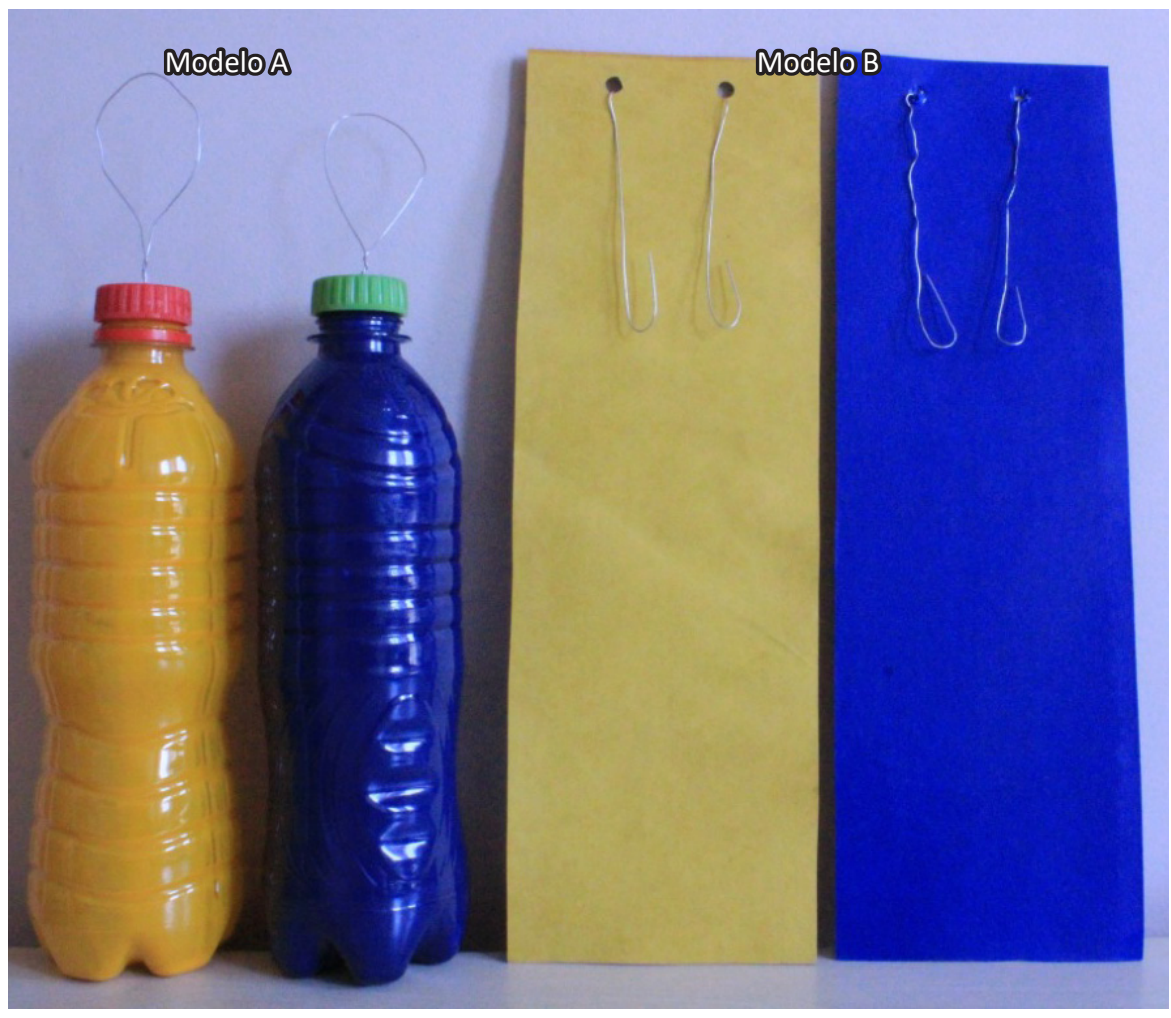

Figura 1. Modelos de armadilhas adesivas artesanais: (A) garrafa PET e (B) papel-cartão, nas colorações amarela e azul-escuro Foto: Janaína Pereira dos Santos

Figure 1. Adhesive handcrafted traps of models: (A) PET bottle and (B) paperboard, in yellow and dark blue colors Photo: Janaína Pereira dos Santos

das em apenas uma face. Para propiciar a captura de insetos em ambos os lados, as folhas foram dobradas e coladas com cola comum. Em seguida, foram cortadas em placas menores, propiciando a confecção de quatro armadilhas com $10 \mathrm{~cm} \times 30 \mathrm{~cm}$ e uma armadilha com $10 \mathrm{~cm} \times 8 \mathrm{~cm}$. Para pendurar as armadiIhas foram feitos dois orifícios na parte superior da placa e, em cada um deles, fixou-se um pedaço de arame fino de aproximadamente $20 \mathrm{~cm}$. A cola entomológica (artesanal ou comercial) foi aplicada com um pincel em ambas as faces da placa.

Cola entomológica artesanal: os ingredientes usados no preparo da cola foram o breu e óleo vegetal. O breu é um produto residual sólido utilizado para a fabricação de colas, obtido de plantas coníferas (Pinus spp.), sendo comercializado em casas agropecuárias no formato de barra. Na preparação da cola, qualquer óleo vegetal pode ser utilizado, porém, o óleo de soja é o mais barato. Desse modo, a cola foi obtida da mistura do breu moído com o óleo de soja, misturando-se os ingredientes em uma panela funda durante cinco minutos sobre fogo baixo, para o derretimento do breu, conforme Santos et al. (2017). Para 100 armadilhas do tipo garrafa PET são necessários em torno de $500 \mathrm{~mL}$ de cola, obtidos da mistura de $400 \mathrm{~g}$ de breu e $200 \mathrm{~mL}$ de óleo de soja; para 100 armadilhas do tipo papel-cartão são necessários em torno de $750 \mathrm{~mL}$ de cola, obtidos da mistura de $600 \mathrm{~g}$ de breu e $300 \mathrm{~mL}$ de óleo de soja (SANTOS et al., 2017). A cola artesanal tem durabilidade de cinco a sete dias, sem a ocorrência de chuva, perdendo a adesividade após esse período. Salienta-se que, logo após a ocorrência de chuva, é necessário reaplicar a cola nas armadilhas.

\section{Custos das armadilhas}

Os dois modelos de armadilhas artesanais, feitos tanto com cola entomológica artesanal quanto comercial, foram 
mais baratos que o modelo de armadiIha comercial.

O custo médio unitário da armadiIha comercial tipo placa adesiva foi de $\mathrm{R} \$$ 3,80. Já os custos médios unitários dos modelos artesanais A (garrafa PET) e B (papel-cartão), utilizando cola entomológica artesanal, foram de $\mathrm{R} \$$ 0,46 e $\mathrm{R} \$$ 0,67, respectivamente (Tabela 1 ). 0 modelo A (garrafa PET + cola artesanal) é 8,2 vezes mais barato que o modelo comercial, enquanto o modelo B (papel-cartão + cola artesanal) é 5,6 vezes mais barato. Salienta-se que o arame e o pincel são materiais que poderão ser reutilizados e, dessa forma, ao se confeccionar mais armadilhas, os valores desses materiais não precisarão ser computados.

Os custos médios unitários dos modelos A e B utilizando cola entomológica comercial foram de $\mathrm{R} \$ 1,50$ e $\mathrm{R} \$ 2,22$, respectivamente (Tabela 2 ). Assim, os modelos artesanais A e $B$, com cola entomológica comercial, são respectivamente 2,5 e 1,7 vezes mais baratos que o comercial. No entanto, no cálculo do custo da cola entomológica comercial não foi computado o valor do frete, pois este dependerá da quantidade adquirida e da região de envio. Portanto, se a cola comercial não for adquirida no comércio local, os custos das armadilhas serão maiores.

Tendo em vista que a troca de armadilhas, em geral, é feita a cada sete dias em função da quantidade de insetos capturados, verifica-se que, utilizandose armadilhas comerciais, o custo será de $\mathrm{R} \$ 380,00$ semana ha $^{-1}$ e $\mathrm{R} \$ 1.628,57$ mês ha-1 (considerando-se um mês composto por 30 dias). Enquanto o custo do modelo mais barato de armadilha artesanal (modelo A + cola artesanal) será de $R \$ 46,55$ semana ha ${ }^{-1}$, representando uma economia de R\$ 1.429,07 mensais ha-1 em comparação ao modelo comercial.

Em regiões com alta pluviosidade e em cultivos extensos a céu aberto, a cola entomológica comercial pode ser uma alternativa para o uso em armadilhas artesanais, pois é insolúvel em água e apresenta durabilidade superior à artesanal. Desse modo, ao se utilizar armadilha artesanal do modelo A com cola entomológica comercial, o custo será de $\mathrm{R} \$ 150,20$ semana $\mathrm{ha}^{-1}$, propor-
Tabela 1. Materiais necessários e custos para a confecção de 100 armadilhas artesanais dos modelos A (garrafa PET) e B (papel-cartão), com cola entomológica artesanal

Table 1. Necessary materials and costs for the confection of 100 handcrafted traps of model A (PET bottle) and B (paperboard), using handcrafted entomological glue

\begin{tabular}{lccc}
\hline Material & $\begin{array}{c}\text { Quantidade para } \\
\mathbf{1 0 0} \text { armadilhas }\end{array}$ & $\begin{array}{c}\text { Valor médio } \\
\text { (R\$)* }\end{array}$ & $\begin{array}{c}\text { Valor médio } \\
\text { unitário } \\
\text { (R\$) }\end{array}$ \\
\hline Tinta & Armadilha modelo A (garrafa PET) & \\
\hline Solvente & $900 \mathrm{~mL}$ & 22,05 & 0,2205 \\
\hline Breu & $150 \mathrm{~mL}$ & 1,75 & 0,0175 \\
\hline Óleo de soja & $400 \mathrm{~g}$ & 17,40 & 0,174 \\
\hline Arame & $200 \mathrm{~mL}$ & 0,95 & 0,0095 \\
\hline Pincel & $20 \mathrm{~m}$ & 1,28 & 0,0128 \\
\hline Total & 1 unidade & 3,12 & 0,0312 \\
\hline \multicolumn{4}{|c|}{ Armadilha modelo B (papel-cartão) } \\
\hline Papel-cartão & 20 folhas & 27,00 & 0,4655 \\
\hline Breu & $600 \mathrm{~g}$ & 26,10 & 0,27 \\
\hline Óleo de soja & $300 \mathrm{~mL}$ & 1,43 & 0,0143 \\
\hline Arame & $40 \mathrm{~m}$ & 2,56 & 0,0256 \\
\hline Pincel & 1 unidade & 3,12 & 0,0312 \\
\hline Cola branca comum & 1 unidade/250g & 6,96 & 0,0696 \\
\hline Total & & $\mathbf{6 7 , 1 7}$ & $\mathbf{0 , 6 7 1 7}$ \\
\hline
\end{tabular}

*Valores cotados em agosto de 2020.

Tabela 2. Materiais necessários e custos para a confecção de 100 armadilhas artesanais dos modelos A (garrafa PET) e B (papel-cartão), com cola entomológica comercial Table 2. Necessary materials and costs for the confection of 100 handcrafted traps of model $A$ (PET bottle) and B (paperboard), using commercial entomological glue

\begin{tabular}{lccc}
\hline Material & $\begin{array}{c}\text { Quantidade } \\
\text { para } \mathbf{1 0 0} \\
\text { armadilhas }\end{array}$ & $\begin{array}{c}\text { Valor médio } \\
(\mathbf{R} \$)^{*}\end{array}$ & $\begin{array}{c}\text { Valor médio } \\
\text { unitário } \\
\text { (R\$) }\end{array}$ \\
\hline Tinta & Armadilha modelo A (garrafa PET) \\
\hline Solvente & $900 \mathrm{~mL}$ & 22,05 & 0,2205 \\
\hline Arame & $150 \mathrm{~mL}$ & 1,75 & 0,0175 \\
\hline Pincel & $20 \mathrm{~m}$ & 1,28 & 0,0128 \\
\hline Cola entomológica & 1 unidade & 3,12 & 0,0312 \\
\hline Total & $1 \mathrm{Kg}$ & 122,00 & 1,22 \\
\hline & Armadilha modelo B (papel-cartão) \\
\hline Papel-cartão & 20 folhas & $\mathbf{2 7 , 0 0}$ & $\mathbf{1 , 5 0 2}$ \\
\hline Arame & $40 \mathrm{~m}$ & 2,56 & 0,27 \\
\hline Pincel & 1 unidade & 3,12 & 0,0312 \\
\hline Cola branca comum & 1 unidade/250g & 6,96 & 0,0696 \\
\hline Cola entomológica & $1,5 \mathrm{Kg}$ & 183,00 & 1,83 \\
\hline Total & & $\mathbf{2 2 2 , 6 4}$ & $\mathbf{2 , 2 2 6 4}$ \\
\hline
\end{tabular}

*Valores cotados em agosto de 2020. 
cionando uma economia de $\mathrm{R} \$ 984,86$ mensais ha-1 em comparação ao uso de armadilhas comerciais.

\section{Recomendações}

Os modelos de armadilhas artesanais desenvolvidos pela Epagri são mais viáveis economicamente do que o modelo comercial do tipo placa adesiva, sendo de 1,7 até 8,2 vezes mais baratos, proporcionando uma economia de até $\mathrm{R} \$ 1.429,07$ mensais ha ${ }^{-1}$.

O uso de armadilhas é uma técnica economicamente viável e ambientalmente segura, podendo ser adotada em cultivos orgânicos e em áreas urbanas. Os modelos feitos com cola artesanal são mais apropriados para ambientes menores e/ou fechados, tais como hortas domésticas, estufas e interior de residências. Para cultivos maiores e a céu aberto (ex.: pomares e lavouras) recomenda-se o uso dos modelos artesanais feitos com cola comercial, pois além de apresentarem custo inferior são tão eficientes e duráveis quanto o modelo de armadilha comercial.

Neste trabalho foram apresentadas várias opções de modelos de armadiIhas. Contudo, a escolha final do modelo partirá do usuário da tecnologia, o qual deverá avaliar o custo e o benefício em função das suas condições financeiras, das condições climáticas da região de cultivo e da disponibilidade de mão de obra e tempo para confeccionar as armadilhas artesanais, podendo, assim, optar pelo modelo de armadilha que mais se adéque às suas reais possibilidades.

\section{Referências}

CILEK, J.E. Attraction of colored plasticized corrugated boards to adult stable flies, Stomoxys calcitrans (Diptera: Muscidae). Florida Entomologist, Gainesville, v.86, n.4, p.420-423, 2003.

CYTRYNOWICZ, M.; MORGANTE, J.S.; SOUZA, H.M.L. Visual responses of south american fruit flies, Anastrepha fraterculus, and mediterranean fruit flies, Ceratitis capitata, to colored rectangles and spheres. Environmental Entomology, Lanham, v.11, n.6, p.1202-1210, 1982.

GAERTNER, C.; BORBA, R.S. Diferentes cores de armadilhas adesivas no monitoramento de pragas em alface hidropônica. Revista Thema, Pelotas, v.11, n.1, p.4-11, 2014.

GUAJARÁ. M.; CARVALHO, A.G.; SANTOS, W.; GONÇALVES, K. Resposta de Euphalerus clitoriae (Hemiptera: Psyllidae) a armadilhas adesivas de diferentes cores. Revista Árvore, Viçosa, v.28, n.1, p.117-120, 2004.

KAVRAN, M.; PETRIĆ, D.; IGNJATOVIĆĆUPINA, A.; ZGOMBA, M. Evaluation of different monitoring methods for Musca domestica L. 1758 (Diptera: Muscidae) indoor population. Contemporary Agriculture, Novi Sad, v.68, n.3-4, p.103-112, 2019.

KOGAN. M. Integrated pest management: historical perspectives and contemporary developments. Annual Review of Entomology, Palo Alto, v.43, n.1, p.243-70, 1998.

MOLINA, R.O.; NUNES, W.M.C.; GONÇALVES, A.M.O.; NUNES, M.J.C.; ZANUTTO, C.A. Monitoramento populacional das cigarrinhas vetoras de Xylella fastidiosa, através de armadilhas adesivas amarelas em pomares comerciais de citros. Ciência e Agrotecnologia, Lavras, v.34, n.spe, p.1634-1639, 2010.

PROKOPY, R.J.; BOLLER, E. Response of European cherry fruit flies to colored rectangles. Journal Economic of Entomology, Lanham, v.64, n.6, p.1444-1447, 1971.

RESENDE, A.L.S.; SILVA, E.E.; GUERRA, J.G.M.; AGUIAR-MENEZES, E.L. Amostragem de pulgões alados utilizando bandeja d'água e placa adesiva. Seropédica, RJ, 2007. 4p. (Circular Técnica, 19).

SANTOS, J.P.; WAMSER, A.F.; BECKER, W.F.; MUELLER, S.; SUZUKI, A. Captura de insetos sugadores e fitófagos com uso de armadiIhas adesivas de diferentes cores nos sistemas de produção convencional e integrada de tomate em Caçador, SC. Horticultura Brasileira (Suplemento), Brasília, v.26. p.157164, 2008.

SANTOS, J.P; ANSILIERO, A.A.; FAGUNDES, E. Confecção de armadilhas artesanais de baixo custo para a captura de insetos-praga. In: Simpósio Internacional de Ciência, Saúde e Território, 4., 2017, Lages. Anais[...] Lages, 2017. p.308-314.

\section{Sigos a Epagrij nos redes socialis}
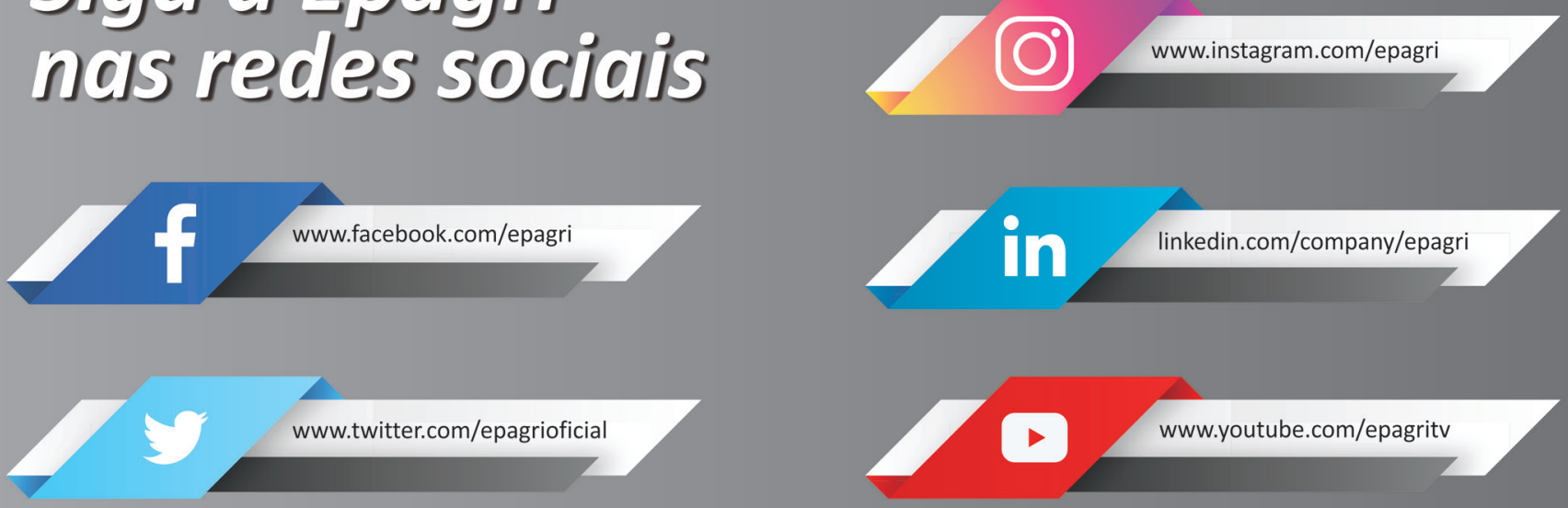